\title{
DESARROLLO DE PRODUCTOS CÁRNICOS INNOVADORES PARA EL CONSUMIDOR: PERCEPCIONES HACIA LAS HAMBURGUESAS DE CORDERO ENRIQUECIDAS CON ADITIVOS NATURALES
}

\author{
Andrés Horrillo*a, Susana García-Torres ${ }^{b}$, Eva Crespo-Cebada ${ }^{a}$, David Tejerinab, Francisco J. \\ Mesíasa $^{a}$ Antonio Rodríguez-Ledesma ${ }^{a}$ Carlos Díaz-Caroc ${ }^{c}$. \\ ${ }^{a}$ Departamento de Economía. Universidad de Extremadura - Ctra. Cáceres s/n;06071 - Badajoz (España, \\ andreshg@unex.es; fimesias@unex.es; ecreceb@unex.es; rledesma@unex.es ).b'Area de Calidad de la \\ carne. CICYTEX Junta de Extremadura. Autovía A5. km 372. 06187. Guadajira. Badajoz (España, \\ susana.garciat@juntaex.es; tejerinabarrado@yahoo.es). ${ }^{c}$ Facultad de Empresariales, Finanzas y Turismo. \\ Universidad de Extremadura. Avda. de la Universidad $s / n$. 10071 Cáceres (España; \\ carlosdiazcar@gmail.com ).
}

\section{Resumen}

El consumo de carne de cordero ha disminuido considerablemente en los últimos años (Rabadán et al., 2020), lo que ha provocado importantes pérdidas para el sector. Esto unido a los cambios en el tipo de alimentación de la población (comida rápida, comida preparada...) ha influido para la posible apertura de nuevos nichos de mercado para la carne de cordero. En el presente trabajo se ha llevado a cabo un estudio a través de la metodología de focus group para valorar las percepciones de los consumidores con respecto a la introducción en el mercado de una hamburguesa de cordero con aditivos naturales (cereza y nuez pecana). Los resultados muestran una elevada frecuencia en el consumo de hamburguesas entre los participantes, el lugar de consumo en los hogares y como principal factor de compra el precio. En cuanto a las hamburguesas propuestas (cordero enriquecidas), los participantes expusieron una reticencia a la carne de ovino, prefiriendo las de vacuno. La mayoría de los participantes mostraron indiferencia hacia los aditivos, aunque, si son naturales como la cereza y la nuez pecana y en la etiqueta se especifica con mayor claridad podrían ser beneficiosos para su venta.

Palabras clave: focus group, investigación cualitativa, carne procesada, hamburguesas de cordero, aditivos naturales.

\section{Introducción}

En los últimos años, el consumo de carne de cordero ha disminuido considerablemente (Rabadán et al., 2020), lo que ha provocado importantes pérdidas en el sector. Esto unido a los cambios en el estilo de vida de la población en los últimos años, los cuales demandan más comida rápida o lista para comer, ha llevado al sector a desarrollar estrategias para diversificar sus productos y estos sean más atractivos para el consumidor, como es el caso de las hamburguesas. Las hamburguesas son productos ampliamente consumidos, debido a su comodidad, bajo coste y agradable sabor. Sólo en España el consumo de hamburguesas supera los 3 millones de unidades semanales (MAPA, 2018). A pesar de ello, también se percibe como un alimento poco saludable, por su relación con la carne roja (Binnie et al., 2014), y por su contenido en grasas saturadas y pobre en fibra, que se asocia con la prevalencia de enfermedades cardiovasculares, cáncer colorrectal y obesidad (Spencer et al., 2005).

Debido a esto, durante los últimos años se han llevado cabo multitud de estudios para contrarrestar y mejorar este aspecto de las hamburguesas. Algunos de estos, han examinado la adicción de antioxidantes para mantener sus propiedades durante el almacenamiento o para mejorar sus beneficios para la salud, como subproductos de tomate (García et al., 2009), subproductos de uva y aceituna (Andrés et al., 2017; SáyagoAyerdi et al., 2009) o aceite esencial (Pateiro et al., 2018), aceite de corteza de canela (Hussain et al., 2021), especias como orégano (Vergara et al., 2020) o la incorporación de fibra vegetal en hamburguesas (García et al., 2009; Hautrive et al., 2019).

En este sentido, la carne de cordero de razas autóctonas (Merina) se considera un producto natural de extraordinarios atributos sensoriales y una mayor concentración de n-3 (Linares et al., 2007, 2008) y su producción está ligada en gran medida al ecosistema dehesa de forma sostenible. Así, la adición de antioxidantes naturales y fibra procedente de recursos naturales, como la cereza o la nuez pecanera, daría lugar a un producto saludable y a priori atractivo para el consumidor.

Estos nuevos productos pueden, desde el punto de vista de productores y transformadores, cubrir un nicho de mercado y ser completamente seguros y satisfactorios tanto higiénica como nutricionalmente, pero los consumidores no tienen por qué compartir esta apreciación. Esto hace necesario conocer las percepciones de los consumidores y esta es una tarea compleja, ya que son numerosos los factores que afectan a las percepciones de los consumidores hacia los alimentos, como por ejemplo la salud, la cultura alimentaria, las tradiciones, etc. Por ello se ha considerado que la técnica de investigación focus group, podía ser un 
enfoque válido para este estudio, ya que es un tipo de investigación cualitativa, flexible y adaptable, y a vez adecuada para descubrir la naturaleza de un problema o para identificar alternativas de acción.

En este contexto, el objetivo de este artículo es explorar las percepciones de los consumidores con relación al uso de aditivos naturales (cereza y nuez pecana) en hamburguesas elaboradas con carne de ovino Merino (raza autóctona de España), con el fin de mejorar sus propiedades nutricionales y mantenerlas durante su conservación. Para ello, y por medio de la técnica del focus group, se han analizado distintos temas como los hábitos de consumo y compra de hamburguesas, los factores que inciden en su compra, la influencia de origen, la especie y raza en la decisión de compra de hamburguesas y la opinión sobre los productos cárnicos procesados enriquecidos.

\section{Metodología}

\subsection{Focus Group}

Un focus group debe estar formado por entre 6 y 12 participantes (Malhotra \& Birks, 2006) para su adecuado desarrollo, y así el moderador no tendrá problemas para llevar la discusión hacia los temas de la investigación. También se recomienda que la composición de los grupos represente la diversidad sociodemográfica de la población estudiada y que el lugar de celebración del grupo de discusión también es relevante (Gaspar et al., 2016).

En esta investigación se celebraron cuatro focus groups durante los meses de marzo y julio de 2020, a las que asistieron un total de 40 participantes (9-12 personas por sesión). Estas sesiones se realizaron en 3 municipios de la región de Extremadura. Los participantes se seleccionaron mediante un muestreo de conveniencia, habitual en la investigación cualitativa (Eldesouky \& Mesias, 2014). La tabla 1, muestra la distribución por genero, edad y nivel de estudios de los participantes en las sesiones.

2.2. Desarrollo de focus group

La sesión se inicia con la explicación del proyecto de investigación en la que se encuadra la actividad (Research Project reference: IB18114) para poner en antecedentes a los participantes. Posteriormente, se inicia el debate siguiendo un guion que se proporciona al moderador y este va introduciendo temas de discusión para que los participantes den su opinión y así sucesivamente, siguiendo el guion. Al terminar cada sesión, se ofrecia a los participantes una degustación de hamburguesas de cordero enriquecidas con nuez pecana y cereza, tras lo que los participantes proporcionaron sus comentarios al respecto. Para su posterior análisis, las sesiones fueron grabadas en video y audio (todos los participantes dieron su consentimiento firmado por escrito). El tiempo total de cada sesión fue de alrededor de 120 minutos de media.

\subsection{Análisis de datos}

Las grabaciones de vídeo y audio de las cuatro sesiones fueron transcritas y anonimizadas para su posterior análisis. El análisis de los datos recogidos se realizó mediante el análisis de contenido (Stewart \& Shamsasani, 2014). El análisis de contenido es una técnica de investigación que busca obtener inferencias válidas y reproducibles a partir de los textos para reducir la cantidad de materiales de entrada (Flick, 2009). Para los fines de este trabajo, inicialmente se procesó la información y se organizó por temas comunes, agrupando bajo cada tema todas las ideas o conceptos que se mencionaron repetidamente durante todas las sesiones. Con el fin de mejorar la robustez de los resultados, se aplicó la triangulación de analistas, procedimiento frecuentemente utilizado en la investigación cualitativa (Eldesouky et al., 2015; Horrillo et al., 2020). Por último, se calculó la frecuencia de mención de cada categoría/concepto como forma de mostrar su importancia relativa, ya que se supone que aquellos ítems que generalmente reciben mayor frecuencia de mención son los más relevantes.

\section{Resultados}

3.1. Frecuencia de consumo y formatos de consumo de las hamburguesas (\% de mención por los participantes).

Las categorías más comentadas en relación con este aspecto son el tipo de hamburguesas consumidas (34\%) y los factores que afectan a la compra (23\%). Más concretamente, entre los tipos de hamburguesas consumidas se observa un amplio número de comentarios, destacando el que hace referencia a su consumo casero, con un $9 \%$ de respuesta, seguido de "diferencia entre caseras e industriales", con $8 \%$. El formato y el origen son comentarios con menor frecuencia junto con "fácil cocinado" y "procesado no favorece su venta".

Otro aspecto que recibe bastantes comentarios es el precio, que se sitúa como uno de los más relevantes factores en la decisión de compra por parte de los consumidores (10\%), junto con el hecho de que los participantes declaran comprar la carne para hamburguesa en carnicería (13\%). Finalmente, respecto a la frecuencia de consumo, los participantes parecen comentar que no suelen consumirlas, y que consideran que el público que más los consume es el de los jóvenes.

\subsection{Influencia del origen, la especie y la raza en la decisión de compra}


Cuadro 1. Influencia del origen, la especie y la raza en la decisión de compra

\begin{tabular}{|c|c|c|}
\hline Categoría & Subcategoría & \% mención \\
\hline Tipo de hamburguesa comprada & $\begin{array}{c}\text { Distinguen si es industrial o hecha en casa } \\
\text { Hamburguesas de cerdo } \\
\text { Hamburguesas de vacuno } \\
\text { No existen productos de ovino y caprino } \\
\text { Hamburguesa de pollo } \\
\text { Hamburguesa Mixta }\end{array}$ & $\begin{array}{c}1 \\
3 \\
15 \\
1 \\
6 \\
6\end{array}$ \\
\hline Importancia de la especie/raza & $\begin{array}{l}\text { Si se fijan en la especie para la compra } \\
\text { SI se fijan en la raza para la compra } \\
\text { No se fijan en la raza para la compra }\end{array}$ & $\begin{array}{c}20 \\
8 \\
5 \\
\end{array}$ \\
\hline $\begin{array}{l}\text { Características organolépticas del } \\
\text { producto }\end{array}$ & $\begin{array}{c}\text { Características organolépticas de la carne de ovino muy particularidades } \\
\text { Probar carnes de otras especies para aceptar su consumo }\end{array}$ & $\begin{array}{l}5 \\
3\end{array}$ \\
\hline Origen territorial & $\begin{array}{c}\text { Si se fijan origen territorial de la carne } \\
\text { No se fijan origen territorial de la carne } \\
\text { Mayor preferencia si las hamburguesas son con carne producida en } \\
\text { Extremadura }\end{array}$ & $\begin{array}{c}11 \\
5 \\
6\end{array}$ \\
\hline Tipo de producción en granja & Se fija en las formas de producción de los corderos & 4 \\
\hline
\end{tabular}

3.3. Opinión de los participantes sobre el conocimiento y la disposición a pagar por los productos cárnicos procesados enriquecidos

Cuadro 2. Conocimiento y la disposición a pagar por los productos cárnicos procesados enriquecidos

\begin{tabular}{ccc}
\hline Categoría & Subcategoría & \% mención \\
\hline Conocimiento & Conocimiento del producto & 27 \\
& Si se fijan en el origen de los aditivos de los aditivos & 23 \\
Aditivos & Preferencia de aditivos naturales & 5 \\
& Necesaria explicación de los aditivos & 4 \\
& Poco saludables (Reticencia al termino aditivos) & 2 \\
Existe publicidad engañosa en aditivos & 10 \\
Mejoras organolépticas y & Varían el sabor original del producto & 2 \\
\hline saludables & El consumo motivado por el sabor y no por beneficios & 2 \\
& saludables & 6 \\
\hline Lugar de consumo & Mejoran las características del producto & 2 \\
\hline & Consumo lúdico-fuera de casa & 6 \\
\hline Disposición a pagar & Si, están dispuestos a pagar por productos enriquecidos & 6 \\
& con aditivos & 2 \\
& Si, están dispuestos a pagar por productos sin aditivos & 2 \\
& Mas información sobre aditivos en los productos mejoraría & la venta \\
& No entienden el sobreprecio por productos con aditivos (en & \\
\hline
\end{tabular}

3.4. Opinión de los participantes sobre el caso particular de la hamburguesa de cordero enriquecida con cereza y nuez pecana

Los asistentes comentaron la existencia de una cierta reticencia de la población al consumo de carne de cordero (9,1\% de las opiniones), debido a que es considerada una carne con sabor fuerte y a la que los consumidores jóvenes no están habituados. En cuanto al lugar de promoción y/o comercialización de estos productos, los participantes tienen una idea clara, la cual queda reflejada en resultados como la venta de este producto en restaurantes especializados (14\%), tiendas especializadas (18\%), no comercializarlo en lineales de grandes supermercados (16\%) y sobre todo se muestra la idea de que los participantes ven a este tipo de producto como gourmet (21\%) y no un producto cotidiano fácil de encontrar en carnicerías o supermercados.

\section{Conclusiones}

Hay una clara preocupación de los consumidores de hamburguesas por el consumo de carnes de calidad y saludables, aspecto que obliga a establecer dichos estándares en la promoción de nuevos productos. La carne de cordero es clasificada como carne roja, pero existe la percepción por parte de los consumidores de que los sistemas de producción bajo condiciones naturales en la que se produce permiten que sea en gran medida considerada como una materia prima saludable.

Según la mayoría de los asistentes a las sesiones de focus group el lugar de mayor consumo de hamburguesas es en el propio domicilio, lo que plantearía si la promoción de estos productos debe ir dirigida primeramente a la venta en establecimientos minoristas, antes que en restaurantes y hamburgueserías. 
Los participantes concluyeron que estarían dispuestos a pagar un precio superior por las hamburguesas de cordero enriquecidas con respecto a las convencionales. Además, si estas disponen de garantías e información sobre el origen y los beneficios que los aditivos aportarían, podrían posicionarse como un atractivo producto para un segmento de la población que busca el consumo de este tipo de alimentos.

La consideración del origen de la carne es un aspecto que atrae el interés de los asistentes, así como la utilización de aditivos de calidad de origen, todo ello en línea con lo que ya se ha comprobado con otros productos de calidad. Según los participantes, esta información debería explicitarse en el etiquetado de los productos para que estuviera disponible para la consulta del consumidor.

\section{Bibliografía}

Andrés, A. I., Petrón, M. J., Adámez, J. D., López, M., \& Timón, M. L. (2017). Food by-products as potential antioxidant and antimicrobial additives in chill stored raw lamb patties. Meat Science, 129, 62-70. https://doi.org/10.1016/j.meatsci.2017.02.013

Binnie, M. A., Barlow, K., Johnson, V., \& Harrison, C. (2014). Red meats: Time for a paradigm shift in dietary advice. Meat Science, 98(3), 445-451. https://doi.org/10.1016/j.meatsci.2014.06.024

Eldesouky, A., \& Mesias, F. (2014). An insight into the influence of packaging and presentation format on consumer purchasing attitudes towards cheese: A qualitative study. Spanish Journal of Agricultural Research, 12(2), 305-312. https://doi.org/10.5424/sjar/2014122-5520

Eldesouky, A., Pulido, A. F., \& Mesias, F. J. (2015). The Role of Packaging and Presentation Format in Consumers' Preferences for Food: An Application of Projective Techniques. Journal of Sensory Studies, 30(5), 360-369. https://doi.org/10.1111/joss.12162

Flick, U. (2009). An introduction to qualitative research (4th ed.). SAGE Publications Ltd.

García, M. L., Calvo, M. M., \& Selgas, M. D. (2009). Beef hamburgers enriched in lycopene using dry tomato peel as an ingredient. Meat Science, 83(1), 45-49. https://doi.org/10.1016/j.meatsci.2009.03.009

Gaspar, P., Escribano, M., \& Mesias, F. J. (2016). A qualitative approach to study social perceptions and public policies in dehesa agroforestry systems. Land Use Policy, 58, 427-436. https://doi.org/10.1016/j.landusepol.2016.06.040

Hautrive, T. P., Piccolo, J., Rodrigues, A. S., Campagnol, P. C. B., \& Kubota, E. H. (2019). Effect of fat replacement by chitosan and golden flaxseed flour (wholemeal and defatted) on the quality of hamburgers. Lwt, 102, 403-410. https://doi.org/10.1016/j.lwt.2018.12.025

Horrillo, A., Gaspar, P., Mesias, F., Elghannam, A., \& Escribano, M. (2020). Understanding the barriers and exploring the possibilities of the organic livestock sector in dehesa agroforestry systems : a multiactor approach for effective diagnosis. Renewable Agriculture and Food Systems, 35(6), 663-677. https://doi.org/https://doi.org/10.1017/S1742170519000334

Hussain, Z., Li, X., Zhang, D., Hou, C., Ijaz, M., Bai, Y., Xiao, X., \& Zheng, X. (2021). Influence of adding cinnamon bark oil on meat quality of ground lamb during storage at $4{ }^{\circ} \mathrm{C}$. Meat Science, $171,108269$. https://doi.org/10.1016/j.meatsci.2020.108269

Linares, M. B., Berruga, M. I., Bórnez, R., \& Vergara, H. (2007). Lipid oxidation in lamb meat: Effect of the weight, handling previous slaughter and modified atmospheres. Meat Science, 76(4), 715-720. https://doi.org/10.1016/j.meatsci.2007.02.012

Linares, M. B., Bórnez, R., \& Vergara, H. (2008). Effect of stunning systems on meat quality of Manchego suckling lamb packed under modified atmospheres. Meat Science, 78(3), 279-287. https://doi.org/10.1016/j.meatsci.2007.06.009

Malhotra, N. K., \& Birks, D. F. (2006). Marketing Research. An Applied Approach.Pearson Education, Harlow.

MAPA. (2018). Informe del consumo de alimentación en España 2017. In Ministerio de Agricultura, Pesca y Alimentación. Gobierno de España. Secretaría General Técnica. Centro de Publicaciones.

Pateiro, M., Barba, F. J., Domínguez, R., Sant’Ana, A. S., Mousavi Khaneghah, A., Gavahian, M., Gómez, B., \& Lorenzo, J. M. (2018). Essential oils as natural additives to prevent oxidation reactions in meat and meat products: A review. Food Research International, 113(April), 156-166. https://doi.org/10.1016/j.foodres.2018.07.014

Rabadán, A., Martínez-Carrasco, L., Brugarolas, M., Navarro-Rodríguez de Vera, C., Sayas-Barberá, E., \& Bernabéu, R. (2020). Differences in Consumer Preferences for Lamb Meat before and during the Economic Crisis in Spain. Analysis and Perspectives. Foods, 9(6), 696. https://doi.org/10.3390/foods9060696 
Sáyago-Ayerdi, S. G., Brenes, A., \& Goñi, I. (2009). Effect of grape antioxidant dietary fiber on the lipid oxidation of raw and cooked chicken hamburgers. LWT - Food Science and Technology, 42(5), 971976. https://doi.org/10.1016/j.lwt.2008.12.006

Spencer, E. H., Frank, E., \& McIntosh, N. F. (2005). Potential effects of the next 100 billion hamburgers sold by McDonald's. American Journal of Preventive Medicine, 28(4), 379-381. https://doi.org/10.1016/j.amepre.2005.01.009

Stewart, D. W., \& Shamsasani, P. N. (2014). Focus groups: theory and practice. SAGE Publications Inc.

Vergara, H., Cózar, A., \& Rubio, N. (2020). Effect of adding of different forms of oregano ( Origanum vulgare ) on lamb meat burgers quality during the storage time. CyTA - Journal of Food, 18(1), 535542. https://doi.org/10.1080/19476337.2020.1794981 